\title{
L'ANTHROPOLOGIE CLINIQUE AU CARREFOUR DES PSYCHOTHÉRAPIES
}

\author{
NICOLAS DURUZ \\ Professeur de psychologie clinique à l'Université de Lausanne \\ Codirecteur de l'Institut Universitaire de Psychothérapie au Département de Psychiatrie - \\ CHUV
}

\section{Correspondance :}

Institut de psychologie

Anthropole - UNIL

Dorigny

$\mathrm{CH}$ - 1015 Lausanne

e-mail : Nicolas.Duruz@unil.ch

\section{RÉSUMÉ}

L'individualisme qui caractérise la société démocratique moderne n'est pas sans conséquence sur le lien social ainsi que sur les institutions qui en sont garantes. Le champ de la psychothérapie n'échappe pas à cette évolution du contexte social qui est le sien, et s'en fait le reflet par une fragmentation toujours plus marquée des approches théoriques, et donc de ses méthodes.

Dans ce contexte de morcellement du champ thérapeutique, la tendance à la spécialisation technique, ainsi qu'à la disqualification mutuelle des différents courants, fait parfois oublier l'objet même de la psychothérapie, à savoir, l'être humain en souffrance.

Plus que jamais, il apparaît donc nécessaire, pour le devenir d'une psychothérapie à visage humain, que tout thérapeute prenne conscience de la portée et de la limite de l'approche à laquelle il prête obédience, relativement aux autres courants : il est ainsi invité au double travail de clarification épistémologique sur les présupposés implicites à sa théorie et méthode, et de distanciation affective à l'égard du courant théorique auquel il est rattaché. L'anthropologie clinique d'inspiration phénoménologique apporte les outils indispensables à un tel travail.

\section{MOTS CLÉS}

Anthropologie clinique - Phénoménologie - Psychothérapie comparée - Epistémologie

\section{CLINICAL ANTHROPOLOGY AT THE CROSSROADS OF PSYCHOTHERAPIES}

\begin{abstract}
Individualism, as a part of modern democratic society, has consequences for the social bond and for the institutions that protect it. Psychotherapy reflects this evolution in the social context through an ever-increasing fragmentation of its theories and methods.

Within the context of the parcelling out of the therapeutic fields, the tendency to technical specialisation and to mutual dismissal among the various schools sometimes overshadows the very object of psychotherapy, namely, suffering human beings.

Therefore the future of humane psychotherapy requires the therapists' ever-greater awareness of the scope and the limits of the approach they pledge allegiance to, relative to other schools. Thus therapists ought to epistemologically clarify their theories and methods' implicit presuppositions and to distance themselves emotionally from their theoretical school.
\end{abstract}


To carry out this assignment phenomenology-inspired clinical anthropology provides essential tools.

\section{KEYWORDS}

Clinical Anthropology - Phenomenology - Comparative Psychotherapy - Epistemology 
Le but de cet exposé est de montrer comment l'anthropologie clinique permet de faire face au phénomène de la pluralité des méthodes psychothérapeutiques, en invitant les psychothérapeutes au dialogue. Dialogue entre eux, mais aussi avec leurs patients, sans lequel l'avenir de la psychothérapie m'apparaît bien menacé.

\section{LA FRAGMENTATION DES SAVOIRS ET DES PRATIQUES}

Teintés d'un certain pessimisme, ces propos s'expliquent en partie par le constat que je fais, non seulement en tant que psychologue clinicien et professionnel de la santé, mais aussi comme citoyen, du processus de fragmentation du champ des savoirs et des pratiques sociales, dont l'effet délétère sur la construction du lien social est avéré. Les manières de vivre aujourd'hui en famille, d'avoir une vie sexuelle, de procréer, d'exercer de nouveaux métiers, pour ne citer que ces exemples, sont des plus variées et ne cessent de se diversifier. Dans le champ de la psychothérapie, inutile de commenter le foisonnement des méthodes existantes à ce jour, phénomène qui s'intensifie aujourd'hui, mais que Carl Gustav Jung signalait déjà en 1930 : «Un regard jeté sur la littérature psychothérapeutique, considérable et confuse, écrivait-il, suffit à corroborer ce fait, non seulement qu'on compte diverses écoles qui récemment encore évitaient anxieusement de se concerter sur le fond, mais il existe également des groupes ou associations qui, telles des cellules, se ferment à tout ce qui n'est pas leur croyance. Il est hors de doute que cet état de choses est un signe indéniable de vitalité [...]. Mais, pour instructif qu'il soit, cet état de choses est peu réjouissant ; et d'autre part, il est peu compatible avec la dignité de la science que la discussion, si nécessaire à son développement, soit entravée par un dogmatisme borné ou par des susceptibilités personnelles $\gg{ }^{1}$.

Ce n'est pas le lieu ici d'interroger les raisons de ce foisonnement des pratiques et du processus de fragmentation sociale qu'il génère. Je souhaite juste signaler une des explications avancées par un sociologue français, Marcel Gauchet [8], dans une perspective d'anthropologie politique, qui m'apparaît tout à fait pertinente, et que des professionnels de la santé ne peuvent pas ne pas prendre en considération, s'ils sont tant soit peu responsables et veulent situer leur action dans le contexte de la société démocratique contemporaine. Gauchet a montré comment le lien social de type démocratique doit se comprendre en fonction de l'évolution des sociétés religieuses primitives vers des sociétés religieuses d'Etat, puis des sociétés d'organisation démocratique. Une telle évolution se caractérise par l'épuisement 
progressif du règne de l'invisible et de la transcendance, propre aux sociétés traditionnelles fondées sur une autorité extérieure à elles-mêmes. Selon Gauchet, en fonction du principe de la souveraineté des individus (organisation démocratique), nous vivons dans une société caractérisée par «l'individualisme démocratique», qui repose sur le droit à la liberté d'expression et de décision de tout individu ou groupe d'individus, auquel est lié le devoir de responsabilité. Dans un tel contexte, les processus identitaires centrés sur l'individualisation, l'autonomie, la singularité ou l'identité différentielle, au détriment de l'appartenance, sont fortement valorisées, générant du même coup une pluralité de pensées, de savoirs et de pratiques sociales, qui tôt ou tard ne peuvent qu'entrer en concurrence et en conflit entre eux. Il s'ensuit un type de lien social relativement menacé.

Cette attaque au lien social d'une démocratie, qui agit finalement contre elle-même, ${ }^{2}$ a comme effet de rendre les humains plus isolés les uns par rapport aux autres, perdus dans la « foule solitaire », selon la célèbre expression de David Riesman, il y a quarante ans. Ne pouvant plus se nourrir de sentiments d'appartenance forts, ils sont en peine de reconnaissance. Poussés à toujours inventer, puisque le « prêt-à-porter » n'est plus de mise, ils cherchent alors à davantage s'affirmer. Certes, on assiste depuis plusieurs décades déjà à des efforts sociaux pour maîtriser ce démembrement, qui représente une atteinte directe à une certaine solidarité de l'être ensemble social, mais chacun de ces mouvements a de la peine à réunir les hommes entre eux, ou le réalise avec des effets pervers importants. Le langage des sciences, cherchant à remplacer les certitudes des discours « au nom de Dieu», se trouve contesté par le relativisme postmoderne. L'arsenal du monde technique, capable de réunir anonymement les humains, contribue en même temps à la production d'un homme unidimensionnel. On peut évoquer les grands rassemblements de foule lors de fêtes sportives ou musicales, mais ce ne sont là que des succédanés passagers. Ou encore les nouveaux montages psycho-religieux, suscités par un certain « retour du religieux » dans une société laïque, mais dont les bricolages ésotériques ont de la peine à épouser des formes institutionnelles largement reconnues.

Le champ de la psychothérapie n'échappe pas à cette logique d'individualisme démocratique, où tôt ou tard la question se pose : comment co-exister ? comment chacun peut-il trouver sa place, pour autant que l'autre la lui reconnaisse?

\section{HYPERSPÉCIALISATION ET DISQUALIFICATION}


Le paysage de la diversité foisonnante des pensées et des pratiques sociales ainsi retracé, j'aimerais mettre en évidence deux de ses conséquences, repérables également dans le champ des méthodes psychothérapeutiques: d'une part, le phénomène d'hyperspécialisation qui entache chaque pratique, et d'autre part, les comportements de disqualification dont font souvent preuve les tenants d'une pratique à l'égard de ceux qui en défendent une autre. Il s'agit là, me semble-t-il, de deux dérives inhérentes à tout processus de fragmentation, et par rapport auxquelles l'anthroplogie clinique peut modestement apporter un correctif. Commençons par décrire brièvement ces deux dérives.

L'hyperspécialisation des méthodes psychothérapeutiques se repère au fait qu'elles ont tendance, en fonction de leur modèle, à perdre contact avec la réalité concrète - l'homme en souffrance psychique - au service de laquelle elles ont été conçues. Il s'ensuit des expertises très techniques, appliquées souvent mécaniquement à des domaines de soin très spécifiques, dans l'ignorance des autres méthodes. Mais cette ignorance de l'autre est une indifférence toute apparente : elle véhicule souvent des attitudes de disqualification à son égard, car cet autre représente une menace pour la mise en œuvre et le développement de sa propre méthode. C'est là un deuxième effet de cet individualisme démocratique. Paradoxalement, l'ouverture démocratique conduit à de l'intolérance. Si celle-ci s'exprime le plus souvent sous des formes subtiles, elle peut se manifester parfois de manière très publique et grossière. Inutile de rappeler les derniers émois en France suscités par la publication du rapport de l'INSERM [12] sur l'évaluation des psychothérapies, ou celle du Livre noir [16], rédigé par des auteurs réunis au nom du courant cognitif-comportemental pour pourfendre le mouvement psychanalytique. Je pense aussi aux conflits parfois rudes autour de la repourvue de chaires de psychiatrie ou de psychologie clinique, aux luttes fratricides menées pour défendre son territoire dans la conquête de maisons d'édition ou de subsides de recherche. Ce sont là autant de manœuvres permettant de s'assurer la clientèle de nombreux patients comme celle de futurs psychothérapeutes en formation, ou encore l'appui de certaines autorités publiques.

La question se pose alors : est-il possible de gérer et de faire valoir socialement la diversité, pour qu'elle ne soit pas une atteinte trop violente au lien social, mais dans le meilleur des cas une manière de l'enrichir en le complexifiant. Je ne peux présenter ici les différents courants dans l'histoire de la psychothérapie, qui ont cherché ou cherchent à limiter cette fragmentation inquiétante des formes de psychothérapie. Les solutions proposées par l'éclectisme pragmatique, l'intégrationnisme, le modèle des facteurs communs non 
spécifiques ou la pensée d'école ${ }^{3}$ sont provisoirement satisfaisantes, mais à moyen terme nous font courir encore davantage le danger des écueils brièvement décrits, à savoir de l'hyperspécialisation technique et de la disqualification. C'est là que l'anthropologie clinique me semble apporter des éléments de réponse qui méritent toute notre attention.

\section{UNE CLINIQUE DE L'HOMME ET NON DE SES FONCTIONS}

Concernant le premier écueil de l'hyperspécialisation, on peut soutenir que l'anthropologie clinique, en insistant sur l'expérience originaire de l'homme au monde, et sur la mise en scène narrative de son existence à partir de sa capacité d'éprouver, de penser et de parler, réagit contre une certaine perception technicisante de l'existence humaine par le savoir psychopathologique. L'anthropologie clinique, dans sa visée la plus simple, veut rappeler qu'il s'agit non d'une clinique des fonctions, mais d'une clinique qui concerne l'homme dans son expérience tout à la fois subjective et intersubjective. Une clinique qui, au sens étymologique du terme, se passe au chevet du malade. Dans ce sens, la clinique, c'est l'art de rencontrer l'autre là où il est et où il souffre de se trouver, souffrant de ne pouvoir s'y retrouver.

Quand l'anthropologie phénoménologique nous invite avec Husserl à retourner aux choses elles-mêmes (zu den Sachen selbst), quand celui-ci en vient à mettre l'accent, à la fin de son œuvre surtout, sur l'expérience de l'homme (Erfahrung) en lien avec la Lebenswelt, le monde de la vie ${ }^{4}$, quand Binswanger distingue «l'histoire intérieure de la vie» des «fonctions vitales » de l'organisme, et qu'il affirme que toute existence est co-existence, plaçant dans la réalité d'une Wirheit (nostrité) le fondement même de notre existence, quand Ricœur insiste sur la dimension narrative de toute identité humaine, et j'en passe, quand on suit tous ces auteurs, n'est-on pas invité à toujours chercher quelque chose d'antéprédicatif, à l'origine de la construction de nos modèles si spécialisés, et à rencontrer l'homme dans son expérience originaire plutôt que l'homme d'une abstraction? L'abstraction de l'homme moyen de la psychopathologie différentielle, celle de l'homme ratomorphique issu du laboratoire, pour reprendre une expression de Bertalanffy, et qui oriente de loin certaines formes de thérapie, celle de l'homme neuronal, comme on tend à le dire aujourd'hui, ou même celle d'un homo psychoanalyticus, tel qu'il peut émerger d'une métapsychologie un peu trop horlogère où les instances psychiques se figent, se substantifient, considérées qu'elles sont comme des instances agentielles. 
Retourner à la chose elle-même - en ce qui nous concerne, à l'homme en souffrance d'humanisation -, de manière à nourrir, rendre vivant, concrétiser ce que les modèles risquent d'aplatir avec leurs dispositifs techniques et hyperspécialisés, voilà une première invitation de l'anthropologie clinique.

\section{DÉCOUVRIR LES LIMITES DE SA MÉTHODE GRÂCE À LA RÉDUCTION PHÉNOMÉNOLOGIQUE}

Mais prenons garde : en parlant ainsi, je pourrais faire preuve de naïveté épistémologique. Mes formulations précédentes sont sujettes à la critique, dans la mesure où elles laisseraient entendre qu'on peut atteindre un « résidu des modèles », qui nous permettrait de nous retrouver en deçà, sur un terrain où nous partagerions des facteurs communs, expression bien connue dans le domaine de la recherche en psychothérapie comparée. Non, soyons clairs. Je pense qu'il n'est pas possible de travailler sans modèle, lequel propose chaque fois un éclairage, une méthode, un chemin qui devient direction (selon l'étymologie même du terme meta-odos), donnant lieu à des modes définis d'intervenir. Ce que l'on peut questionner toutefois, c'est la relation à son modèle - celui-là plutôt qu'un autre - modèle toujours porté implicitement par des présupposés ou a priori impliquant des valeurs personnelles et idéologiques. En effet, je fais l'hypothèse que les méthodes psychothérapeutiques se différencient entre elles en fonction de différences encore plus fondamentales que celles déjà contenues explicitement dans leur modèle théorique et leurs techniques. Cette pluralité de méthodes serait donc en partie redevable à des présupposés différents qui organisent leur modèle et la pratique psychothérapeutique en découlant, chaque fois à partir d'une certaine vision de l'homme, de la société ou de la science, pour ce qui est du domaine de la psychothérapie. Ces prémisses ou présupposés organisateurs peuvent s'entendre au sens de Bateson comme « un ensemble d'hypothèses ou de prémisses habituelles, implicites dans la relation entre l'homme et son environnement $»^{5}$. Ils fonctionnent comme des a priori épistémologiques implicites, qui interviennent inévitablement dans l'appréhension et la construction de la réalité, et qui ont pour caractéristique de se valider par eux-mêmes en engendrant un effet de croyance, voire d'évidence.

En explicitant les présupposés qui nous lient à notre modèle, ne pourrions-nous pas alors mieux découvrir ses limites, reconnaître ainsi plus facilement le modèle des autres, en 
d'autres termes éviter partiellement le second écueil de la disqualification dont j'ai parlé ? Je pense que l'anthropologie clinique, particulièrement le courant phénoménologique, est ici d'une aide précieux, en nous proposant une méthode spécifique, celle de la réduction phénoménologique, en vue de dégager les présupposés organisant ou contextualisant chaque méthode psychothérapeutique. C'est là le deuxième apport de l'anthropologie clinique.

Pour le lecteur non familier de la phénoménologie, clarifions cette méthode. Il s'agit d'une opération de la pensée qui vise à suspendre, à mettre entre parenthèses, à jeter le doute (époché) sur l'attitude «naturelle » que nous avons envers la réalité, attitude qui nous fait oublier que c'est à travers un regard spécifique sur elle qu'elle nous apparaît telle. Collés à nos modèles, nous avons tendance à «naturaliser » nos connaissances. Husserl essaie de montrer que toute connaissance du monde s'acquiert dans l'acte transcendental d'une constitution de sens, où la conscience incarnée, à partir des synthèses passives qu'elle opère et qui l'ouvrent à l'horizon de ce qu'elle appréhende, produit un acte originaire où le monde est « thématisé ». Comme l'écrit Giudicelli de manière un peu savante pour les non philosophes, mais combien rigoureuse : «Le monde de la vie dont nous partons toujours, et où nous revenons sans cesse, est antéprédicatif. C'est dans cet entrelacement que se structure, dans l'intentionnalité, l'intelligible le plus complexifié, par esquisses constantes et enrichissement de sens. La recherche de l'explication est cette tension qui prend appui sur le renvoi constant des synthèses passives à l'acte originaire où la subjectivité dans son choix primordial avec la totalité du monde comme donné, s'ébranle dans la genèse active. La recherche de l'explication est ce dépassement de la synthèse passive en synthèse active, qui devient synthèse prédicative. [...] Dans l'activité antéprédicative gît une préconstitution passive de signification dont la subjectivité constituante incarnée dans le monde de la vie est imprégnée $»^{6}$. En parlant de la suspension des thèses métaphysiques sur le monde, de celles de l'empirisme ou du rationalisme, du subjectivisme ou de l'objectivisme, d'une approche naturaliste ou historiciste, ou encore d'une conception causaliste ou téléologique du monde, Husserl en 1927 écrit : «On trouve partout des motifs légitimes, mais partout des demimesures ou des façons irrecevables de rendre absolues des vues unilatérales qui ne sont légitimes que d'un point de vue relatif et abstrait » ${ }^{7}$. L'époché consiste donc à mettre entre parenthèses ses préjugés d'analyse, à revenir à sa propre subjectivité constituante, dans ce moment originaire de la constitution de sens. Selon la belle expression d'Husserl, il s'agit « de faire vœu de pauvreté en matière de connaissance» pour ne pas s'aliéner dans des modèles trop absolus. 
Selon une perspective surtout constructionniste, dans laquelle la phénoménologie ne veut certes pas se laisser enfermer, on est donc amené à penser que nos modèles psychothérapeutiques sont toujours porteurs d'une connaissance d'avant la connaissance, laquelle organise notre regard sur la vie, l'infléchit inexorablement en nous faisant voir certains aspects plutôt que d'autres, générant par-là même certaines évidences sur notre manière de saisir les troubles psychiques.

Dans deux publications plus particulièrement, qui relatent les résultats d'une étude empirique $[5,6]$, j'ai cherché à expliciter ces présupposés à l'œuvre dans les différentes orientations thérapeutiques, présupposés qui sont de l'ordre de l'implicite et résistent toujours à se laisser formuler. Selon que l'on conçoit l'être humain comme un individu capable de maîtrise et de contrôle, par exemple, ou plutôt en fonction de sa singularité subjective et historique ou de ses appartenances, ou encore selon un point de vue plus holistique que fonctionnel, etc., il est évident que l'on aura davantage d'affinités avec telle ou telle orientation psychothérapeutique.

Par exemple, présenter la psychothérapie comme une technique capable de résoudre des problèmes vécus par un individu présuppose 1. que la psychothérapie est d'abord une affaire de technique qui peut être administrée par un thérapeute non impliqué, 2. que la vie humaine consiste avant tout en un problème à résoudre et 3. qu'il faut aider des individus souffrant de problèmes personnels; alors que d'autres présupposeront 1. que la psychothérapie est essentiellement une expérience relationnelle, 2. que la vie est quelque chose qu'il faut vivre le mieux possible et que nous devons endurer sans qu'il n'y ait de solutions et 3. que les patients souffrent de plus en plus d'une société malsaine, certaines prises en charge trop individualisantes risquant dans ce sens de faire le jeu de cette société.

\section{SCIENCE ET CROYANCE}

A ce stade de la réflexion, je souhaiterais souligner que l'époché, cet acte de prise à distance ou de désabsorption de l'objet captatif, opéré par l'arrêt du flux continuel des pensées et des activités, est une opération difficile, car loin d'être une seule entreprise d'ordre cognitif. Elle engage notre affectivité, la passion de nos préjugés, que Montesquieu qualifiait comme ce qui fait que je m'ignore moi-même plutôt que ce qui fait que j'ignore des choses. 
Selon moi, nos préjugés ou présupposés ne s'assimilent pas à de simples costumes du soir, qu'on peut mettre et déposer sans autre. Ils ressortissent à des croyances entendues dans une acception non cognitiviste, dans le sens qu'ils ne peuvent être conçus comme des représentations en attente d'arbitrage, devant faire l'objet d'une preuve scientifique. Ils sont plutôt traversés par la passion de nos idéaux, source d'intolérance et d'ostracisme.

Une croyance nous engage toujours. Si nous y tenons, c'est parce qu'elle nous tient dans notre identité. Nous y engageons ce qui nous est le plus cher, au plus près de l'étymologie du verbe anglais to believe, qui dans le vieil anglais $\mathrm{du} \mathrm{XVI}^{\mathrm{e}}$ siècle signifiait aimer, chérir, considérer comme cher, ce même sens que l'on retrouve dans le mot allemand belieben, et selon la même racine du mot latin libet (il plaît) ou libido (plaisir). Une croyance qui nous met au service de, et qui ne porte pas d'abord sur un jugement d'existence, comme on l'entend souvent aujourd'hui, dans l'expression par exemple croire en Dieu. C'est dans ce contexte que Bacon pouvait définir en 1625 la croyance du vrai comme «jouissance » par opposition au questionnement ou à la recherche de la vérité. On est loin du rationalisme critique de Popper, au nom duquel il pensait pouvoir neutraliser les croyances en préconisant l'échange entre scientifiques qui doivent se soumettre aux procédures de falsifiabilité.

Dans le monde plus restreint de la psychothérapie, Mc Dougall, cette grande psychanalyste, était très lucide lorsqu'elle écrivait. « Nous avons besoin de nous assurer qu'il existe un ordre dans le chaos du fonctionnement psychique et qu'il y a des théories pour exliquer les changements psychiques. [...] En nous attachant à une école théorique, nous faisons partie d'une famille, nous sommes moins seuls face aux incertitudes qui nous assaillent tous les jours. [...] L'idéal serait de tenir dans le même respect que les nôtres, poursuivait-elle, les théories des autres ; cela nous permettrait de mieux percevoir les limites de nos propres modèles et leur prégnance sur nous $\gg{ }^{8}$.

\section{SE DÉCOUVRIR DIFFÉRENT GRÂCE À L’AUTRE}

Mais comment avoir accès à ses croyances, comment travailler sur elles pour ne pas en être trop dupe, tout en sachant qu'elles nous traversent et nous constituent au plus profond de notre identité. Idéalement, il serait souhaitable, à un niveau métathéorique, de pouvoir faire l'inventaire de ses croyances, qui engagent toujours une certaine conception de l'homme, de la société et de la vérité scientifique. Cela rencontrerait sans doute le vœu formulé par Bateson à 
l'égard des scientifiques: «Il est évidemment souhaitable (mais non absolument nécessaire) que l'homme de science soit conscient de ses présuppositions, et qu'il soit capable de les formuler. Pour pouvoir porter un jugement scientifique, de toute façon, il est recommandé, pour ne pas dire indispensable, de connaître les présuppositions des collègues faisant des recherches dans le même domaine $»^{9}$.

Il s'agirait en quelque sorte d'établir une «charte épistémologique », cherchant à faire l'inventaire de nos présupposés. Ce travail utile en soi risque toutefois d'être insuffisant, parce que se limitant à un exercice encore trop cognitif et réalisé de manière individuelle. Je pense que c'est dans l'interaction directe entre collègues, au niveau d'un échange sur nos pratiques, comme nous allons l'expliquer plus bas, que nous parviendrons le mieux à prendre conscience des prémisses et croyances qui nous animent. Le réel dialogue entre psychothérapeutes repose selon moi sur le repérage par chacun d'eux de ses présupposés, compatibles pour certains, incompatibles pour d'autres, avec ceux de ses collègues, ce qui permet à chacun de situer sa méthode dans une articulation épistémologique à celles des autres.

C'est à ce niveau que se situe le troisième apport de l'anthropologie clinique, dans sa dimension herméneutique, comme l'ont développé entre autres Gadamer et Ricœur. Pour l'anthropologie herméneutique, tout travail d'élucidation de soi passe par la médiation de l'autre. Il n'y a pas d'immédiateté à soi-même. "Nous ne sommes que ténébres à nousmêmes, écrivait Malebranche, il faut que nous nous regardions hors de nous pour nous voir ». Husserl ne laissait-il pas entendre que l'Ego transcendental n'est ni formellement, ni matériellement dans la conscience, il est dehors, dans le monde, c'est un être du monde, comme l'Ego d'autrui ?

Dans ce sens, pour favoriser le travail d'articulation épistémologique différentielle entre les méthodes psychothérapeutiques, je propose la constitution de groupes de psychothérapeutes d'orientations diverses, conduits par un modérateur et dans lesquels chacun s'engage à respecter la pensée et le travail des autres. Dans le but de rencontrer d'autres manières de pratiquer, chacun est alors invité à présenter à tour de rôle une situation thérapeutique en cours, où il se trouve dans l'impasse. Les commentaires, réactions et suggestions des collègues, à travers leurs propres propositions d'hypothèses et de stratégies 
thérapeutiques différentes suscitées par le modérateur, doivent favoriser la prise de conscience de présupposés différents, concernant la manière de se représenter la santé et le bonheur de l'être humain par exemple, de concevoir la fonction du temps et de l'argent dans le processus thérapeutique, des manières différentes de se référer à un modèle thérapeutique, de se définir comme psychothérapeute dans la société actuelle, etc. Autant de comparaisons qui permettraient progressivement de découvrir la diversité des présupposés en jeu, parfois convergents, parfois incompatibles. Et aussi, il faut le souhaiter, d'en modifier certains. ${ }^{10}$

On pourrait dire que nous entrons là dans le domaine d'une anthropologie clinique, à visée comparative, qui a cette exigence que dès le moment où l'on s'intéresse à l'autre différent de soi, on se trouve transformé dans la compréhension de soi-même. En d'autres termes, la connaissance comparée des autres, c'est simultanément la mise en perspective de soi-même. La plupart d'entre nous appartenons à une culture psychothérapeutique spécifique, qui s'est forgé sa propre langue, ce qui n'empêche pas certains d'avoir fait l'expérience d'autres cultures, dont ils ressortent habituellement enrichis dans leur manière de vivre la leur. Ce n'est pas pour autant une invitation à rechercher un esperanto culturel. On ne peut jamais renoncer à sa langue maternelle; elle nous enracine dans notre identité première. Mais à fréquenter d'autres langues, on peut découvrir davantage les finesses et la complexité de la sienne propre, voire l'enrichir. Goethe, cet esprit universel, nous invite à cette ouverture sans renier l'ancrage identitaire de la langue-mère, lui qui disait : "Wer nur eine Sprache kennt, kennt keine », ce qui pourrait se traduire en ces termes : «Celui qui ne sait qu'une langue, n'en connaît aucune »!

La rencontre avec son patient, on l'a compris, implique pour l'anthropologie clinique une rencontre avec un autre soi-même, un Mitmensch avec qui nous partageons le même projet d'un travail d'humanisation. Mais il importe également, comme on vient de le voir, que nous appartenions aussi à une même communauté de langue, proches dans la manière d'appréhender le dysfonctionnement psychique et sa transformation, de vivre la relation thérapeutique, ou encore de «faire partie » de la société. Très vite, des incompréhensions de base peuvent s'installer entre le psychothérapeute et le patient qui ne partagent pas du tout la même vision du monde. Il est donc indispensable que le psychothérapeute vérifie qu'un minimum d'affinités électives existe entre lui et son patient quant à leur «charte épistémologique » respective. 
La clinique, comme le soutient l'anthropologie clinique, est l'art des repossibilisations. Ne faut-il pas aussi plusieurs méthodes psychothérapeutiques pour donner la chance à tout homme au visage figé par une souffrance excessive de retrouver l'expression d'une vie humainement possible ? Des repossibilisations dont aucune orientation n'a le monopole, mais au service desquelles chacune peut faire valoir sa contribution. 


\section{RÉFÉRENCES}

1. Bateson G. 1971. La cybernétique du « soi »: une théorie de l'alcoolisme. In Bateson G. (ed). 1977. Vers une Ecologie de l'Esprit. Tome I. Paris : Seuil, 225-252.

2. Bateson, G. 1979. La nature et la pensée. Trad. fr. 1984. Paris : Seuil.

3. Duruz N. 1994. Psychothérapie ou psychothérapies? Prolégomènes à une analyse comparative. Neuchâtel et Paris : Delachaux et Niestlé.

4. Duruz N. 2006. Faut-il en finir avec la pluralité des orientations en psychothérapie ? Psychothérapies $26: 69-77$.

5. Duruz N., Lob R. 1996. Qu'est-ce que la psychothérapie ? Regard métaclinique de 77 psychothérapeutes suisses. Psychothérapies 16 : 171-180.

6. Duruz N., Lob R. 1997. Psychothérapeutes : analyse de trois présupposés. Psychothérapies $17: 67-77$.

7. Duruz N., Gennart M. 2002. Traité de psychothérapie comparée. Genève : Médecine et Hygiène.

8. Gauchet M. 1985. Le désenchantement du monde. Une histoire politique de la religion. Paris : Gallimard.

9. Gauchet M. 2003. La démocratie contre elle-même. Paris : Gallimard.

10. Giudicelli S. 2004. L’implication phénoménologique comme fondement scientifique à la psychothérapie des psychoses. Perspectives Psy 43 :101-106.

11. Husserl E. 1927. Psychologie phénoménologique. Trad. fr. 2001. Paris : J. Vrin.

12. INSERM 2004. Psychothérapie. Trois approches évaluées. Expertise collective. Institut National de la Santé et de la Recherche Médicale.

13. Jung C. G. 1934. La névrose et l'auto-régulation psychologique. Situation de la psychothérapie en 1930. In Jung C. G. (ed). 1953. La guérison psychologique, Genève : Ed. Georg \& Cie.

14. McDougall J. 1988. Quelles valeurs pour la psychanalyse ? Revue Française de Psychanalyse 52 : 585-612.

15. Merleau-Ponty M. 1945. Phénoménologie de la perception. Paris : Ed. Gallimard. 16. Meyer C. (dir.). 2005. Le livre noir de la psychanalyse. Paris : Ed. des Arènes.

\footnotetext{
${ }^{1}$ Jung [13], p. 189.

${ }^{2}$ Gauchet [9]

${ }^{3}$ Duruz [4]
} 
${ }^{4}$ Ce que Merleau-Ponty prolongera par son insistance sur une subjectivité incarnée, qui fait que c'est notre corps vécu tout entier qui est esprit et que c'est à partir de lui que nous sommes au monde. «Il n'y pas d'homme intérieur, écrit-il, l'homme est au monde, c'est dans le monde qu'il se connaît. Quand je reviens à moi à partir du dogmatisme de sens commun ou du dogmatisme de la science, je trouve non pas un foyer de vérité intrinsèque, mais un sujet voué au monde ». Merleau-Ponty [15], p.V.

${ }^{5}$ Bateson [1], p. 230.

${ }^{6}$ Giudicelli [10], p. 103.

${ }^{7}$ Husserl [11], p. 243.

${ }^{8}$ Mc Dougall [14], p. 606.

${ }^{9}$ Bateson [2], p. 131.

${ }^{10}$ Duruz et Gennart [7], pp. 8-10. 